\title{
Finding a novel interacting protein of the hepatic carcinoma related gene MIP: NF-kB essential modulator (NEMO)
}

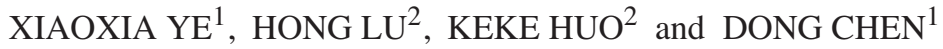 \\ ${ }^{1}$ Department of Histology and Embryology, Guangdong Medical College, Zhanjiang 524023, Guangdong Province; \\ ${ }^{2}$ State Key Laboratory of Genetic Engineering, Institute of Genetics, School of Life Sciences, \\ Fudan University, Shanghai 200433, P.R. China
}

Received June 21, 2010; Accepted August 24, 2010

DOI: $10.3892 /$ or_00001065

\begin{abstract}
MafF interacting protein (MIP) is product of a candidate gene related to liver cancer development and progression. Here, we demonstrated that MIP could inhibit the proliferation of cancer cells through colony formation assays, and the inhibition ability of MIP to SMMC7721 cells was notably stronger than to HeLa cells. Using pGBKT7-MIP as bait, a human placenta cDNA library was screened using yeast two-hybrid system and a middle fragment of the NF-кB essential modulator (NEMO) was obtained as a novel important MIP interacting protein fragment, which contained 228 amino acid sequence from the 120 to 347 residue. Then the full coding sequence of NEMO was amplified from Clontech Placenta Marathon cDNA library and yeast mating assay verified the interaction of MIP and full length NEMO in yeast. GST pull-down assay and co-immunoprecipitation assay confirmed that MIP bound to NEMO specifically and directly. These results indicated that MIP interacted with NEMO and suggested that MIP could be involved in NF- $\mathrm{B}$ signaling pathway, which is helpful to clarify the inhibition function of MIP to cancer cell proliferation.
\end{abstract}

\section{Introduction}

Cancer related gene MIP (GeneID: 727764) was cloned through chromosome structure comparing between normal liver tissue and hepatic carcinoma tissue, subtractive hybridization and large scale colony formation screening by Shanghai Cancer Institute (1). Earlier studies showed that MIP could inhibit the growth of cancer cells and growth rate decreased by about $50 \%$ when MIP was transfected into HeLa cells. It has been found that MIP could interact with phosphatidylinositol-four-phosphate adaptor protein-1 (FAPP1) associated protein FASP1 in cytoplasm (2). FAPP1

Correspondence to: Dr Xiaoxia Ye, Department of Histology and Embryology, Guangdong Medical College, Zhanjiang 524023, Guangdong Province, P.R. China

E-mail: yemissing@yahoo.com.cn

Key words: MafF interacting protein, yeast two-hybrid, NEMO/ $\mathrm{IKK} \gamma, \mathrm{NF}-\kappa \mathrm{B}$ pathway specifically recognized and bound to the $\mathrm{PH}$ domain of PtdIns4P that was a key member in phosphoinositide cycle, but did not bind to other members (3). Through series interactions of MIP-FASP1-FAPP1-PtdIns4P, MIP may be involved in phosphoinositide metabolism and some associated signaling pathways. In 2006, another study reported that MIP could interact with bZip transcription factor MafF (v-maf musculoaponeurotic fibrosarcoma oncogene homolog F) in the nucleus (4). Being a member of Maf transcription factor family, MafF possesses a conserved basic region and a heptad repeat motif of leucines, which are responsible for DNA binding and interaction with other regulator proteins, respectively (5). However, MafF lacks trans-active domain and can not activate transcription alone (6). MIP functions as a co-activator of MafF and they together can activate transcription of target gene OTR. Hence it was named as MafF interacting protein (MIP) (4).

These studies as above show that MIP can interact with a number of proteins with multiple complex functions, such as signaling transduction and transcription regulation. However, the exact mechanism on how MIP inhibits the growth of cancer cells is not yet clear. To investigate more potential roles of MIP on cell proliferation and growth, a yeast two hybrid screening was performed and another important MIPinteracting protein fragment, the middle fragment of NF- $\mathrm{BB}$ essential modulator (NEMO) was found. Then this novel specific interaction between MIP and full length NEMO protein was investigated. We report the results of our initial studies suggesting that MIP was probably involved in NF-кB signaling pathway, which provides helpful clues to explain MIP's inhibition function to the proliferation of cancer cells and upstream regulation of the $N F-\kappa B$ pathway.

\section{Materials and methods}

Plasmid construction. The full length ORF of MIP was amplified from human placenta cDNA library (Clontech) using sense primer 5'-CGGAATTCATGCTCTGCCCTAGA GCCG-3' and anti-sense primer 5'-GCGTCGACTCACTGG TAGCCAGCCA-3'. The full coding sequence of NEMO was amplified from Placenta Marathon cDNA library (Clontech) using sense primer 5'-CCGAATTCATGAATAGGCACCT CTGGAAG-3' and anti-sense primer 5'-GCCTCGAGCTA 

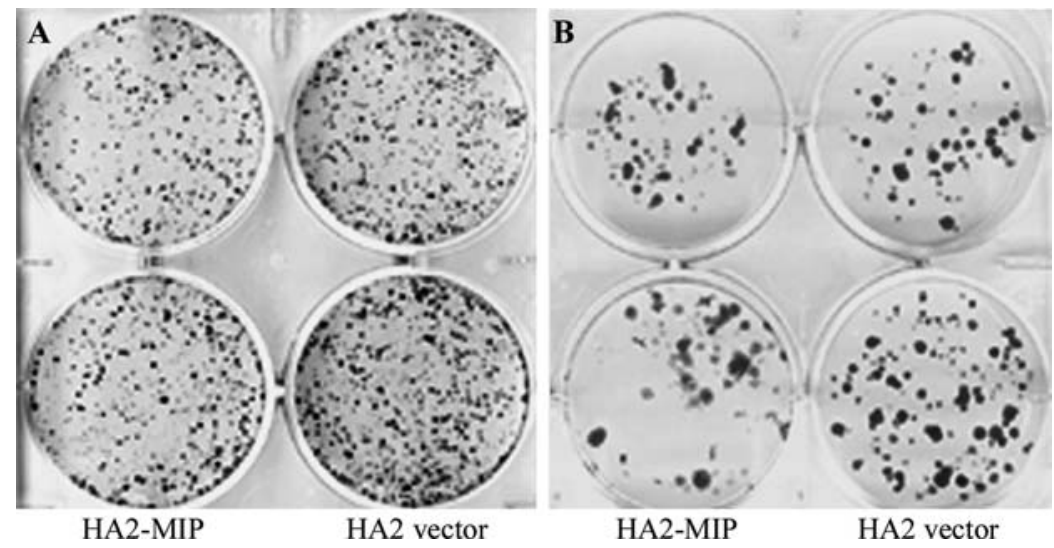

Figure 1. The colony formation assays of MIP in carcinoma cells. The colony numbers were counted and relative colony formation efficiency of HA2-MIP transfected cells were calculated by comparing with the colony numbers of HA2 vector transfected cells. (A) The relative colony formation efficiency of MIP was 64\% in HeLa cells. (B) The relative colony formation efficiency of MIP reached $78 \%$ in SMMC7721 cells.

CTCAATGCACTCCATGAC-3'. The plasmids including pGBKT7-MIP and pGADT7-NEMO for yeast assay, pGEX5X-1-MIP and pGADT7-NEMO for binding assay in vitro, pCMV-HA2-MIP and pCMV-Myc-NEMO for mammalian cellular assay, were constructed by standard molecular biology techniques and confirmed by DNA sequencing.

Colony formation assay. Human hepatocellular carcinoma SMMC-7721 cells and human cervical carcinoma HeLa cells were cultured in DMEM (Gibco, Life Techologies Inc., NY) with $10 \%$ FBS (PAA Laboratories $\mathrm{GmbH}$ ) in a 6-well plate. When cells grew to $60-70 \%$ confluence, they were transfected with pCMV-HA2-MIP or pCMV-HA2 void vector by Lipofectamine 2000 (Invitrogen), respectively. The transfected cells were incubated in DMEM supplemented with $10 \%$ FBS and $400 \mu \mathrm{g} / \mathrm{ml} \mathrm{G} 418$ until untransfected cells of control were killed entirely and then turned to DMEM medium containing 10\% FBS and $200 \mu \mathrm{g} / \mathrm{ml} \mathrm{G} 418$ until colonies appeared. Two parallel assays were performed for each sample. The colonies were stained by crystal violet. The colony numbers of different samples and cell lines were compared.

Yeast two-hybrid screening. pGBKT7-MIP was used as the bait to screen a human placenta cDNA library in Clontech Matchmaker Two-hybrid system 3. More than $1 \times 10^{6}$ transformants were screened on SD-Trp-Leu-Ade-His medium with $5 \mathrm{mM}$ 3-aminotriazole (SD-4+3AT medium). Obtained clones were tested by $ß$-galactosidase activity assay. Prey plasmids were isolated and re-transformed into yeast along with either pGBKT7-MIP or pGBKT7 to verify the specific interaction. Finally the positive prey plasmids were sequenced and then analyzed.

Yeast mating assay. pGADT7-NEMO was transformed into AH109a yeast. pGBKT7 and pGBKT7-MIP were separately introduced into $\mathrm{Y} 187 \alpha$ yeast. The AH109a yeast harboring AD-NEMO was mated with Y187 $\alpha$ yeast harboring BD-MIP or BD as control, respectively. The obtained diploid yeast strains were tested for reporter gene activities by incubating in SD-Trp-Leu-Ade-His medium containing $5 \mathrm{mM} 3 \mathrm{AT}$ and colony-lift filter assay.
In vitro binding assay. pGEX-5X-1 and pGEX-5X-1-MIP were transformed into Escherichia coli strain BL21. Both GST and GST-MIP were expressed and purified according to the manufacturer's protocol (Amersham Pharmacia Biotech). Using pGBKT7-NEMO plasmid as template, Myc-NEMO fusion protein was generated by the TNT T7 Quick Coupled Transcription/Translation System (Armersham Pharmacia Biotech) and detected by Western blot analysis with antiMyc antibody (Clontech). The GST-MIP or GST bound on glutathione-Sepharose 4B beads were incubated with $10 \mu \mathrm{l}$ translated Myc-NEMO mixture for $4 \mathrm{~h}$ at $4^{\circ} \mathrm{C}$. Then the beads were washed three times with buffer H $(20 \mathrm{mM}$ HEPES, pH 7.7, $50 \mathrm{mM} \mathrm{KCl,} \mathrm{20 \%} \mathrm{glycerol,} \mathrm{0.1 \%} \mathrm{NP-40} \mathrm{and} \mathrm{0.007 \%}$ B-mercaptoethanol) and then boiled in $20 \mu 1$ elution buffer. The bound proteins were separated by $15 \%$ SDS-PAGE and then transferred to PVDF membrane. Membranes were blocked with non-fat milk, then immunolotted with mouse anti-Myc primary antibody and HRP-conjugated rabbit anti-mouse IgG (Calbiochem). The Myc-tagged protein was visualized with SuperSignal West Femto Maximum sensitivity substrate for HRP (Pierce).

Co-immunoprecipitation assay. HeLa cells were cultivated to $70 \%$ confluence and co-transfected with pCMV-Myc-NEMO and pCMV-HA2-MIP, or pCMV-HA2 as negative control. After $48 \mathrm{~h}$, cells were washed with ice-cold PBS and lysed with lysis buffer (Roche) at $4^{\circ} \mathrm{C}$ for $20 \mathrm{~min}$. Cell lysates were pre-cleaned with protein $\mathrm{A} / \mathrm{G}$ Plus agarose beads for $1 \mathrm{~h}$ at $4^{\circ} \mathrm{C}$, then incubated with $200 \mathrm{ng}$ anti-HA antibody at $4^{\circ} \mathrm{C}$ overnight. The beads were gently washed with lysis buffer. The precipitated proteins were eluted by elution buffer and then subjected to Western blot analysis.

\section{Results}

To explore the effect of MIP on carcinoma cells, human hepatocellular carcinoma SMMC-7721 cells and human cervical carcinoma HeLa cells were both used to colony forming assays. As shown in Fig. 1, the colony number was significantly reduced in HA2-MIP transfected cells compared with HA2 vector transfeted cells in both HeLa cells and SMMC7721 cells. Moreover, the proliferation inhibition of 

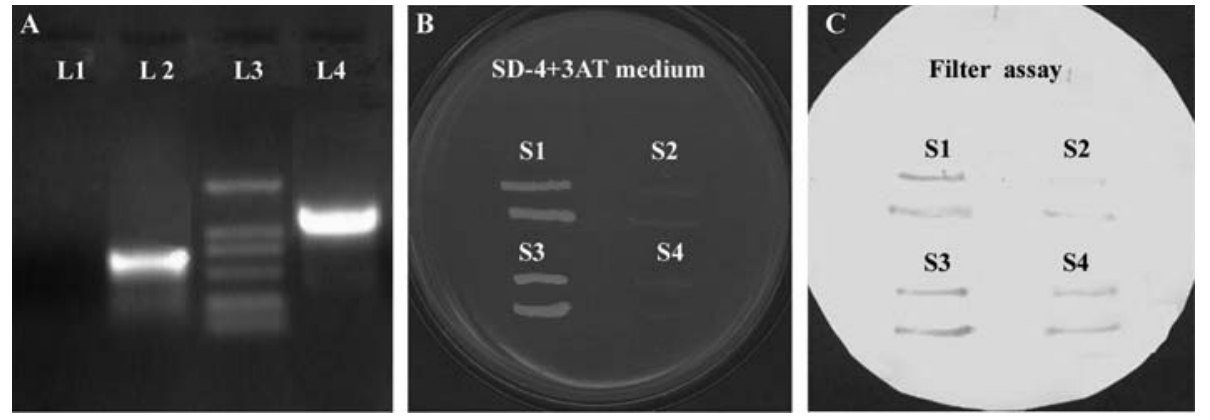

Figure 2. The yeast mating assay of MIP and full length NEMO. (A) The full coding sequence of NEMO was amplified from Clontech Placenta Marathon cDNA library. L1, negative; L2, The cDNA in prey plasmid was 684 bp encoding a middle fragment (from the 120 to 347 amino acids) of NEMO; L3, DL2000 marker; L4, the full coding sequence of NEMO was 1257 bp encoding 419 amino acids. (B and C) The mating diploid yeast strains were tested the reporter genes Ade, His and LacZ by incubating on SD-4+3AT medium and colony lift filter assay. S1, BD-MIP+AD-NEMO; S2, BD+AD-NEMO; S3, BD-p53+AD-T-antigen, positive control; S4, BD-LamC+AD-T-antigen, negative control.
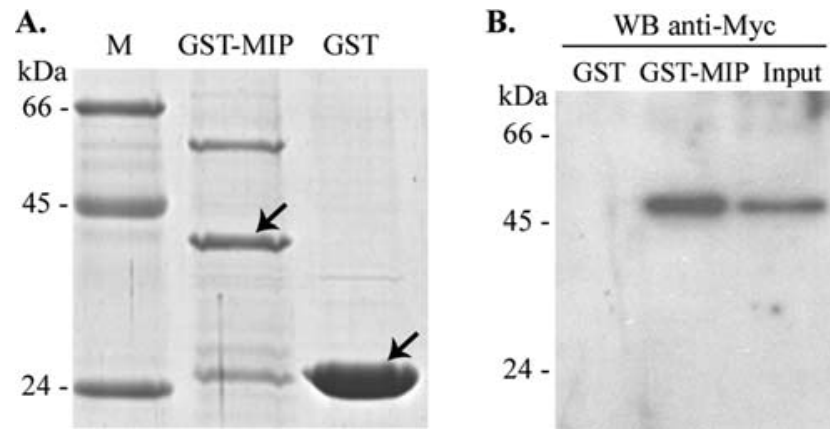

Figure 3. The interaction of MIP and NEMO in vitro. (A) Coomassie staining of purified GST-MIP and GST (indicated with arrows) with the molecular weight of about 43 and $29 \mathrm{kDa}$, respectively. (B) GST pull-down assay in vitro. Myc-NEMO was detected from bound proteins of GST-MIP with anti-Myc antibody, but was not in GST alone. Input was a control using $3 \mu 1$ translated mixture in vitro.

MIP on SMMC-7721 was more apparent than on HeLa cells. Comparing the colony number formed by void vector transfencted cells, the relative colony forming efficiency of MIP in HeLa cell was 64\% (Fig. 1A), while it reached $78 \%$ in SMMC7721 cells (Fig. 1B). These results clearly indicated that the overexpression of MIP inhibited the growth of cancer cells and the inhibition ability of MIP on SMMC7721 cells was stronger than on HeLa cells.

To investigate the possible mechanism of MIP inhibition in cancer cell proliferation, yeast two-hybrid screening was performed. A human placenta cDNA library was screened and the obtained positive prey plasmids were sequenced. The sequence analysis revealed that the inserted cDNA in two prey plasmids was $684 \mathrm{bp}$ (Fig. 2A, lane 2) and encoded a middle protein fragment of the NF- $\mathrm{NB}$ essential modulator (NEMO), which included 228 amino acid sequence from the 120 to 347 residue. To verify the interaction between MIP and full-length NEMO, the full coding sequence of NEMO was amplified from Placenta Marathon cDNA library, which contained $1257 \mathrm{bp}$ (Fig. 2A, lane 4) and encoded 419 amino acids. The full-length NEMO was cloned into pGADT7 plasmid and transformed into Y187 yeast. Then yeast mating assay was performed. As shown in Fig. 2B, the diploid yeast strain containing BD-MIP and AD-NEMO activated the reporter genes Ade and His, and grew on SD-4+3AT medium

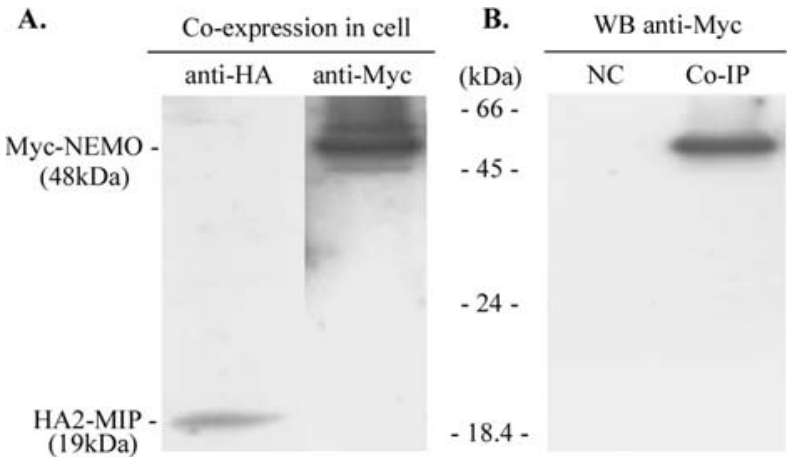

Figure 4. The interaction of MIP and NEMO in mammalian cells. (A) HA2-MIP and Myc-NEMO were expressed in co-transfected cells. (B) Co-immunoprecipitation in vivo. Anti-HA antibody and protein A/G agarose were added into cell lysates to precipitate. The immunoprecipitation of HA2-MIP resulted in co-immunoprecipitation of Myc-NEMO. NC was the negative control using cell lysate that co-transfected with HA2 vector and Myc-NEMO.

(Fig. 2B, S1), while the control yeast strain containing BD and AD-NEMO could not (Fig. 2B, S2). Colony-lift filter assay indicated that LacZ reporter gene was activated in the BD-MIP+AD-NEMO yeast strain, but was not activated in the BD+AD-NEMO yeast strain (Fig. 2C). These results indicated that MIP and NEMO could interact and active reporter genes. The yeast mating assay verified that MIP could specifically bind to full-length NEMO.

Subsequently, GST pull-down assay in vitro was performed to confirm the interaction observed in yeast cells. After induced expression, both GST-MIP and GST were highly expressed in BL21 (Fig. 3A). Fusion protein MycNEMO was translated in vitro using the rabbit reticulocyte lysate and assessed the capability to form a stable complex with GST-MIP or GST alone as the negative control. In Fig. 3B, GST-MIP efficiently and specifically pulled down Myc-NEMO protein, but GST alone did not. GST pull-down assay verified that MIP could specifically bind to NEMO in vitro directly.

To further verify whether MIP interacts with NEMO in mammalian cells, co-immunoprecipitation assays were performed and the results are shown in Fig. 4. The bands of Fig. 4A indicated that HA2-MIP and Myc-NEMO were both expressed in co-transfected cells. When anti-HA antibody 
and protein $\mathrm{A} / \mathrm{G}$-agarose were added into the cell lysates to precipitate HA2-MIP, Myc-NEMO protein was coprecipitated and detected by Western blot analysis using antiMyc antibody (Fig. 4B, lane 2). The cell lysates that cotransfected with pCMV-HA2 and pCMV-Myc-NEMO were used as control and Myc-NEMO was not detected (Fig. $4 \mathrm{~B}$, lane 1). The in vivo binding assay indicated MIP could specifically bind to NEMO in mammalian cells.

\section{Discussion}

MIP was cloned as a candidate gene related to liver cancer development and progression (1). In this study, we proved that MIP could inhibit the proliferation of cancer cells, and this inhibition ability of MIP to SMMC7721 cells is notably stronger than to HeLa cells. These results further confirmed the relationship between MIP and hepatic carcinoma and suggested that MIP was a hepatic carcinoma related gene.

In order to explore the mechanism of MIP inhibition function in cancer cells, we performed a yeast two-hybrid screening and found a novel important MIP interacting protein, a middle fragment of NEMO. The yeast mating assay, in vitro and in vivo binding assays validated the interaction between MIP and full length protein of NEMO. NEMO plays a pivotal role in the activation of the canonical $\mathrm{NF}-\kappa \mathrm{B}$ signaling cascade pathway. In resting cells, NF- $\mathrm{B}$ is kept inactive in the cytoplasm by binding to the inhibitor IкB. In response to various stimuli such as viral and bacterial infections, proinflammatory cytokines and so on, IкB is rapidly phosphorylated by $\mathrm{I} \kappa \mathrm{B}$ kinase complex (IKK) and subsequently ubiquitinated for proteasomal degradation. NF$\kappa \mathrm{B}$ then dissociates from IкB, translocates into nucleus and activates the transcription of various target genes $(7,8)$. The key element in this process is the IKK complex, composed of two catalytic subunits named IKK $\alpha$ and IKK $\beta$, and a regulator subunit IKK $\gamma$. IKK $\gamma$ is critical in the assembly and activity regulation of IKK complex and NF- $\kappa \mathrm{B}$ activation $(9,10)$. For example, a synthetic NEMO-binding domain (DBD) oligopeptide, which exists in IKK $\alpha$ and IKKß, can compete against NEMO to bind with IKK and to inhibit the $\mathrm{NF}-\kappa \mathrm{B}$ activation $(11,12)$. Hence IKK $\gamma$ is also named as the $\mathrm{NF}-\kappa \mathrm{B}$ essential modulator (NEMO). A number of proteins have been identified as directly interacting with NEMO and modulating the activity of IKK complex, including A20, cylindromatosis protein, COP9 signalosome (CSN), zinc finger protein 216 , and protein phosphatase $2 \mathrm{~A}(13,14)$. NEMO can bind the IKK complex with different upstream activators to transfer different upstream signals $(15,16)$. Hence the different NEMO partners are a key issue for upstream regulation induced by different signals in NF-кB pathways.

In our study, MIP was identified as another novel NEMO-interacting protein and involved in the $\mathrm{NF}-\kappa \mathrm{B}$ signaling pathway. It might serve as a specific upstream signaling molecule or a competitor against other activators through binding with NEMO, which results in activating or inhibiting IKK complex activity, and ultimately down- or upregulation of $N F-\kappa B$ activation. $N F-\kappa B$ possesses a bidirectional modulation in apoptosis and oncogenesis (17).

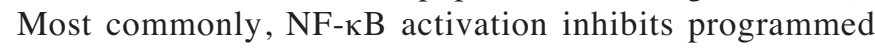

cell death. The expression and activation of $\mathrm{NF}-\kappa \mathrm{B}$ are abnormal in many carcinoma tissues and cell lines, which induce the expression of a number of genes whose products inhibit apoptosis, including cellular inhibitors of apoptosis (c-IAPs), Bcl-2, TNFR associated factor (TRAF1) and TRAF2, A1. On the other hand, in some cases NF-кB activation can induce apoptosis because it activates tumorrepressor gene such as p53, C-Myc, caspase-1, and FasL $(17,18)$. Because of the anti-apoptotic and pro-apoptotic functions of NF- $\mathrm{B}$, upstream regulation induced by different signals is very pivotal for life or death (19). We think that MIP bound to NEMO is involved in NF- $\mathrm{B}$ signaling pathway, which probably resulted in the proliferation inhibition of MIP to cancer cells. These findings demonstrated an important role of MIP and offer useful insight into the NEMO/NF-кB signaling pathway. In-depth study of this interaction, in particular on the effects on cell growth, apoptosis process and NF- $\mathrm{KB}$ activity, will provide a strong basis to clarify the mechanism of MIP inhibition in cancer cells and further explain the relationship between oncogenesis and NF- $\mathrm{\kappa B}$.

\section{Acknowledgements}

This study was supported by Medical Scientific Research Foundation of Guangdong Province, China (No. B2009192) and Scientific Research Foundation of Guangdong Medical College (No. XB1010).

\section{References}

1. Wan D, Gong Y, Zhang P, et al: Large-scale cDNA transfection screening for genes related to cancer development and progression. Proc Natl Acad Sci USA 101: 15724-15729, 2004.

2. Ye XX, Lu H, Ding N, et al: Pp5644 interaccts with phosphatidylinositol-4-phosphate adaptor protein-1 associated protein-1. Mol Cell Biochem 271: 151-158, 2005.

3. Dowler S, Currie RA, Campbell DG, Deak M, Kular M, Downes CP and Alessi DR: Identification of pleckstrinhomology-domain-containing proteins with novel phosphoinositide-binding specificities. Biochem J 351: 19-31, 2000.

4. Ye X, Li Y, Huang Q, et al: The novel human gene MIP functions as a co-activator of hMafF. Arch Biochem Biophys 449: 87-93, 2006

5. Dlakic M, Grinberg AV, Leonard DA and Kerppola TK: DNA sequence-dependent folding determines the divergence in binding specificities between Maf and other bZIP proteins. EMBO J 20: 828-840, 2001.

6. Fujiwara KT, Kataoka K and Nishizawa M: Two new members of the maf oncogene family, mafK and mafF, encode nuclear b-Zip proteins lacking putative trans-activator domain. Oncogene 8: 2371-2380, 1993.

7. Karini M: How NF-кB is activated: the role of the IкB kinase (IKK) complex. Oncogene 18: 6867-6874, 1999.

8. Karin $M$ and Ben-Neriah Y: Phosphorylation meets ubiquitination: the control of NF- $\mathrm{KB}$ activity. Annu Rev Immunol 18: 621-663, 2000.

9. Solt LA, Madge LA and May MJ: NEMO-binding domains of both IKK $\alpha$ and IKK $\beta$ regulate IкB kinase complex assembly and classical NF- $\mathrm{kB}$ activation. J Biol Chem 284: 27596-27608, 2009.

10. Yamaoka S, Courtois G, Bessia C, et al: Complementation cloning of NEMO, a component of the IкB kinase complex essential for NF-кB activation. Cell 93: 1231-1240, 1998.

11. May MJ, D'Acquisto F, Madge LA, Glockner J, Pober JS and Ghosh S: Selective inhibition of NF-кB activation by a peptide that blocks the interaction of NEMO with the IкB kinase complex. Science 289: 1550-1554, 2000. 
12. Aqou F, Courtois G, Chiaravalli J, et al: Inhibition of NF-кB activation by peptides targeting $\mathrm{NF}-\kappa \mathrm{B}$ essential modulator (nemo) oligomerization. J Biol Chem 279: 54248-54257, 2004.

13. Shifera AS: Proteins that bind to IKK $\gamma$ (NEMO) and downregulate the activation of NF- $\mathrm{\kappa B}$. Biochem Biophys Res Commun 396: 585-589, 2010.

14. Shifera AS: Protein-protein interactions involving IKK $\gamma$ (NEMO) that promote the activation of NF-кB. J Cell Physiol 223: $558-561,2010$

15. Markris C, Roberts JL and Karin M: The carboxyl-terminal region of IкB kinase $\gamma(\mathrm{IKK} \gamma)$ is required for full IKK activation. Mol Cell Bio 22: 6573-6581, 2002.
16. Chariot A, Leonardi A, Muller J, Bonif M, Brown and Siebenlist U: Association of the adaptor TANK with the IкB kinase (IKK) regulator NEMO connects IKK complexes with IKK epsilon and TBK1 kinases. J Biol Chem 277: 37029-37036, 2002.

17. Luo JL, Kamata $\mathrm{H}$ and Karin M: IKK/NF- $\mathrm{KB}$ signaling: balancing life and death, a new approach to cancer therapy. J Clin Invest 115: 2625-2632, 2005.

18. Karin $\mathrm{M}$ and Lin A: NF-кB at the crossroads of life and death. Nat Immunol 3: 221-227, 2002.

19. Shishodia S and Aggarwal BB: Nuclear factor- $\mathrm{B}$ activation: a question of life or death. J Biochem Mol Biol 35: 28-40, 2002. 